\title{
Intervenção Coronária Percutânea no Hospital Vera Cruz de Campinas: Resultados de Cinco Anos de Experiência
}

\author{
Silvio Gioppato ${ }^{1}$, Marcelo J. C. Cantarelli', Thomas Borges Conforti ${ }^{1}$, Helio J. Castello Jr. ${ }^{1}$,

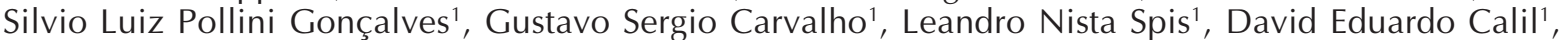 \\ Mauricio T. Nicastro ${ }^{1}$, Pedro A. P. Conforti ${ }^{1}$, Antenor Baucchi ${ }^{1}$, Vitorio Verri', Guilherme C. Verri ${ }^{1}$, \\ Mayara Brunheroto Lourenço', Carlos Eduardo Sampaio', Fabio Rainha Lopes', Mauricio Marson Lopes', \\ Ronaldo Padovani ${ }^{1}$, Thiago Jair Vedovello ${ }^{1}$, Nyder Rodrigues Otero ${ }^{1}$
}

\section{RESUMO}

Introdução: A intervenção coronária percutânea (ICP) tem importante papel no tratamento da doença arterial coronária, em decorrência do desenvolvimento tecnológico e da ampliação de sua utilização nos cenários clínicos de maior complexidade. Apresentamos a experiência de serviço de hemodinâmica que iniciou sua atividade há cinco anos, localizado em hospital em Campinas (SP). Métodos: De 2006 a 2011, 518 pacientes foram submetidos consecutivamente a ICP no Hospital Vera Cruz e incluídos neste estudo. As características clínicas, angiográficas e do procedimento e os resultados clínicos hospitalares foram analisados. Resultados: A média de idade dos pacientes foi de $65,8 \pm 12,2$ anos, $73,5 \%$ eram do sexo masculino, $29,9 \%$ eram diabéticos e metade dos procedimentos foi realizada na vigência de síndrome coronária aguda. No total, foram tratadas 890 lesões (74\% delas do tipo B2/C), sendo implantado 1,6 stent/paciente, $37,1 \%$ dos quais farmacológicos. $\mathrm{O}$ diâmetro e o comprimento do stent foram, respectivamente, de 3,01 $\pm 0,53 \mathrm{~mm}$ e $23,2 \pm 7 \mathrm{~mm}$. O sucesso do procedimento foi de 93,6\%, óbito ocorreu em 2,9\% dos pacientes, acidente vascular cerebral em $0,8 \%$, infarto do miocárdio em 3,5\%, ICP de urgência em $0,8 \%$, e trombose de stent em $0,97 \%$. Hipertensão arterial [odds ratio (OR) 4,48, intervalo de confiança de 95\% (IC 95\%) 1,32-15,16; $\mathrm{P}=0,016$ ] e diabetes (OR 7,24, IC 95\% 1,69-31,05; $\mathrm{P}=0,008)$ foram preditores independentes de eventos adversos cardíacos e cerebrovasculares maiores (ECCAM) hospitalares. Conclusões: $O$ sucesso do procedimento e a baixa ocorrência de ECCAM demonstram a efetividade e a segurança da ICP no atendimento de pacientes da prática clínica diária tratados em um novo serviço de cardiologia intervencionista, inserido em hospital localizado fora da capital do Estado de São Paulo.

DESCRITORES: Angioplastia. Stents. Doença das coronárias.

\section{ABSTRACT \\ Percutaneous Coronary Intervention at the Vera Cruz Hospital in Campinas: Results of a Five-Year Experience}

Background: Percutaneous coronary intervention ( $\mathrm{PCl}$ ) plays an important role in the treatment of coronary artery disease, as a result of technological development and its wide application in different clinical scenarios of greater complexity. We present data from a cath lab that started its activity five years ago at a private hospital in the city of Campinas (SP). Methods: From 2006 to 2011, 518 consecutive patients were submitted to $\mathrm{PCl}$ at the Vera Cruz Hospital and were included in this study. Clinical, angiographic and procedural characteristics and the in-hospital clinical outcomes were analyzed. Results: Patient average age was $65.8+12.2$ years, $73.5 \%$ were male, $29.9 \%$ were diabetics and half of procedures were performed during an acute coronary syndrome. A total of 890 lesions (74\% B2/C type) were treated with 1.6 stents/patient, $37.1 \%$ of them were drug eluting stents. Stent diameter and length were $3.01 \pm$ $0.53 \mathrm{~mm}$ and $23.2+7 \mathrm{~mm}$, respectively. The procedure success was $93.6 \%$, death occurred in $2.9 \%$ of the patients, stroke in $0.8 \%$, myocardial infarction in $3.5 \%$, reintervention in $0.8 \%$ and stent thrombosis in $0.97 \%$. Hypertension [odds ratio $(\mathrm{OR}) 4.48,95 \%$ confidence interval $(95 \% \mathrm{Cl})$ 1.32-15.16; $P=0.016]$ and diabetes $(O R$ 7.24, 95\% Cl 1.69-31.05; $\mathrm{P}=0.008)$ were independent predictors of in-hospital major adverse cardiac and cerebrovascular events (MACCE). Conclusions: The overall procedural success and low incidence of MACCE demonstrated the efficacy and safety of $\mathrm{PCI}$ in the daily clinical practice in a new interventional cardiology service, localized in a hospital outside the capital of the state of São Paulo.

KEY-WORDS: Angioplasty. Stents. Coronary disease.

\footnotetext{
1 Hospital Vera Cruz - Campinas, SP, Brasil.

Correspondência: Silvio Gioppato. Rua Onze de Agosto, 495 Centro - Campinas, SP, Brasil - CEP 13013-100

E-mail: gioppato@terra.com.br

Recebido em: 27/9/2011 • Aceito em: 7/12/2011
} 
A intervenção coronária percutânea (ICP) tem assumido papel de destaque cada vez maior no tratamento da doença arterial coronária, em decorrência do contínuo desenvolvimento tecnológico e da ampliação de sua utilização nos vários cenários clínicos. ${ }^{1}$ Com resultados impactantes sobre a mortalidade no infarto agudo do miocárdio (IAM) e na qualidade de vida nos pacientes mais estáveis, essa modalidade terapêutica tem sido aplicada de maneira crescente no Brasil, onde as doenças cardiovasculares são responsáveis por $30 \%$ de todos os óbitos. ${ }^{2-4}$

A complexidade de um laboratório de hemodinâmica, no entanto, aliada ao alto custo dos equipamentos e insumos e à necessidade de estar inserido em um hospital estruturado para atendimento terciário, com unidade de terapia intensiva e serviço de cirurgia cardiovascular, restringem a oferta da ICP em larga escala em nosso País. Entretanto, o crescimento econômico brasileiro nos últimos 10 anos tem impactado positivamente a atuação do setor de serviços no Brasil em diversas áreas, inclusive a da saúde. Dessa forma, novos hospitais e serviços de hemodinâmica têm surgido em diferentes pontos do País.

Neste estudo apresentamos os dados da experiência em ICP do serviço de hemodinâmica de um hospital privado da cidade de Campinas (SP), que iniciou suas atividades há cinco anos, demonstrando os resultados hospitalares do procedimento nesse cenário contemporâneo.

\section{MÉTODO}

\section{Pacientes}

Em setembro de 2006 foi inaugurado o Serviço de Hemodinâmica e Cardiologia Intervencionista do Hospital Vera Cruz em Campinas (SP). Até agosto de 2011, 518 pacientes foram consecutivamente tratados com ICP e incluídos neste estudo. Foram analisadas as características clínicas, angiográficas e relacionadas ao procedimento, os resultados clínicos e as complicações intra-hospitalares do procedimento. Todas as informações foram coletadas e armazenadas prospectivamente em banco de dados informatizado, desenvolvido e personalizado em parceria com empresa especializada. Todos os pacientes assinaram o termo de consentimento informado para utilização dos dados em apresentações e publicações científicas.

\section{Procedimento}

As intervenções foram realizadas seguindo normas e padrões técnicos vigentes. ${ }^{5}$ Habitualmente os pacientes receberam heparina não-fracionada, na dose de 70-100 Ul/kg no início do procedimento. Nos casos eletivos, os pacientes eram mantidos com dose de $100 \mathrm{mg} / \mathrm{dia}$ de ácido acetilsalicílico e $75 \mathrm{mg} / \mathrm{dia}$ de clopidogrel, com dose de ataque de 300 mg na vés- pera do procedimento. Nos casos urgentes e que não estavam em uso de antiagregantes plaquetários, era administrada dose de ataque de $300 \mathrm{mg}$ de ácido acetilsalicílico e de $600 \mathrm{mg}$ de clopidogrel imediatamente antes, durante ou logo após o procedimento, e nessa situação a utilização de um inibidor da glicoproteína IIb/IIla era obrigatória. Nos procedimentos realizados por via femoral, as bainhas introdutoras foram retiradas 4 horas após a última dose de heparina; nos casos realizados por via radial, a retirada foi imediata ao final do procedimento. A técnica de hemostasia consistiu da combinação de compressões mecânica e manual em todos os casos.

\section{Análise angiográfica}

As análises angiográficas foram realizadas on line em duas ou mais projeções ortogonais, por operadores experientes, após a administração de mononitrato de isossorbida na dose mínima de $20 \mathrm{mg}$ por via intracoronária. As lesões foram classificadas de acordo com os critérios do American College of Cardiology/American Heart Association. ${ }^{6} \mathrm{O}$ diâmetro de referência do vaso foi definido como a média dos diâmetros dos segmentos considerados angiograficamente normais, proximal e distal à lesão. O porcentual do diâmetro da estenose foi definido como [1-(diâmetro mínimo da luz/diâmetro de referência do vaso) x 100]. O comprimento da lesão foi medido como a distância entre as bordas proximal e distal do segmento acometido, sendo consideradas longas as lesões com comprimento $\geq 20 \mathrm{~mm}$. O fluxo antes e após o procedimento foi quantificado de acordo com a classificação TIMI. $^{7}$

\section{Definições}

O sucesso do procedimento foi definido como obtenção de sucesso angiográfico (estenose residual $<30 \%$ no caso de implante de stent e $<50 \%$ no caso de angioplastia com balão, combinado a fluxo TIMI 3 no final do procedimento) e ausência de eventos cardiovasculares e cerebrovasculares adversos maiores (ECCAM), compreendendo óbito de qualquer causa, acidente vascular cerebral (AVC), infarto do miocárdio associado ao procedimento, ICP ou cirurgia de revascularização miocárdica (RM) de emergência. ${ }^{5}$ Os eventos clínicos foram contabilizados no período intra-hospitalar. Infarto do miocárdio associado ao procedimento foi definido pela elevação da creatina quinase fração $M B(C K-M B)>3$ vezes o limite superior da normalidade ou $>5$ vezes quando o valor basal estava alterado, e pelo desenvolvimento de novas ondas $\mathrm{Q}$ patológicas no eletrocardiograma de 12 derivações, em pelo menos duas derivações contíguas. O AVC era confirmado por exame clínico ou métodos de imagem. A ICP ou a RM de emergência foram consideradas quando indicadas por insucesso ou complicação do procedimento índice. 


\section{Análise estatística}

Os dados foram analisados em programa estatístico SPSS versão 15.0. As variáveis contínuas foram expressas em média \pm desvio padrão e as variáveis categóricas, em números absolutos e percentis. Para comparar as variáveis contínuas por ocorrência de desfechos clínicos (ECCAM) foi utilizado o teste $t$ de Student e para as variáveis categóricas, o teste qui-quadrado. Modelos de regressão logística simples e múltipla foram aplicados para identificar preditores de ECCAM. Entraram no modelo de regressão múltipla as seguintes variáveis: idade, sexo, índice de massa corporal (IMC), tabagismo, diabetes, hipertensão arterial sistêmica, dislipidemia, AVC prévio, IAM prévio, RM prévia, insuficiência renal crônica, lesöes B2/C, e extensão da doença coronária (uni vs. multiarterial). Foi adotado o nível de significância de $P<0,05$.

\section{RESULTADOS}

Incluímos nesta análise os 518 pacientes iniciais e consecutivos tratados em nosso serviço, que foram submetidos a 565 intervenções percutâneas (Tabela 1). Predominaram pacientes do sexo masculino (73,5\%), com média de idade de $65,8 \pm 12,2$ anos, e um quarto dos pacientes tinha idade $\geq 75$ anos. A maioria (92,8\%) apresentava IMC > $25 \mathrm{~kg} / \mathrm{m}^{2}$ e 29,9\% eram diabéticos, dos quais quase um quarto utilizava insulina.

TABELA 1 Dados demográficos

\begin{tabular}{|c|c|}
\hline Variável & $n=518$ \\
\hline Idade, anos & $65,8 \pm 12,2$ \\
\hline Idade $\geq 75$ anos, n (\%) & $149(28,8)$ \\
\hline Sexo masculino, n (\%) & $380(73,5)$ \\
\hline Hipertensão arterial, n (\%) & $375(72,4)$ \\
\hline Diabetes melito, n (\%) & $155(29,9)$ \\
\hline Em uso de insulina & $37(23,9)$ \\
\hline Dislipidemia, n (\%) & $302(58,3)$ \\
\hline História de tabagismo, n (\%) & $288(55,6)$ \\
\hline Ativo & $107(20,7)$ \\
\hline Prévio & $181(34,9)$ \\
\hline $\mathrm{IMC}, \mathrm{kg} / \mathrm{m}^{2}$ & $27,4 \pm 4,5$ \\
\hline $\mathrm{IMC} \geq 25$ e $<30, \mathrm{n}(\%)$ & $361(69,7)$ \\
\hline$I M C \geq 30$, n (\%) & $120(23,2)$ \\
\hline AVC prévio, n (\%) & $26(5)$ \\
\hline IM prévio, n (\%) & $129(24,9)$ \\
\hline RM prévia, n (\%) & $118(22,8)$ \\
\hline ICP prévia, n (\%) & $152(29,3)$ \\
\hline
\end{tabular}

AVC = acidente vascular cerebral; ICP = intervenção coronária percutânea; IMC = índice de massa corporal; $\mathrm{n}$ = número de pacientes; RM = cirurgia de revascularização miocárdica.
Metade dos pacientes $(50,4 \%)$ foi tratada no contexto das síndromes coronárias agudas (SCAs), distribuídas entre síndrome coronária aguda sem supradesnivelamento do segmento ST (SCASST) com 58,6\%, IAM com supradesnivelamento do segmento ST (IAMCST) com 37,2\%, e angina pós-infarto com 4,2\%. Quase três quartos dos pacientes com SCASST (72,5\%) tinham escore de risco TIMI médio/alto e 58,7\% estavam em uso de ácido acetilsalicílico nos últimos 7 dias. Entre os pacientes com IAMCST, 12,3\% estavam em classe Killip III/IV (Tabela 2).

Foram abordados 876 vasos (1,7 vaso/paciente), com um total de 890 lesões tratadas. As artérias coronárias direita e descendente anterior foram tratadas em igual proporção $(33 \%)$, houve franca prevalência das lesões complexas tipo B2/C (74\%), e as lesões em bifurcação representaram $11,2 \%$ da amostra, predominando o padrão 1,1,1 da classificação de Medina (56\%) (Tabela 3).

Foram implantados 846 stents (relação de 1,6 stent/ paciente), com sucesso angiográfico sendo atingido em 99,6\%. O diâmetro médio dos stents implantados foi de 3,01 $\pm 0,53 \mathrm{~mm}$ e o comprimento médio, de 23,2 \pm 7 mm, com taxa de utilização dos stents farmacológicos de 37,1\% (Tabela 4).

Sucesso do procedimento foi alcançado em 93,6\% das intervenções, com taxas de óbito hospitalar de $2,9 \%$, AVC de $0,8 \%$ e infarto do miocárdio de 3,5\% (Tabela 5). ICP de urgência foi necessária em 4 casos $(0,8 \%)$, todos por trombose aguda, e nenhum paciente necessitou RM de urgência. A incidência global de trombose foi de $0,97 \%$, sendo $0,77 \%$ de trombose aguda e 0,19\% de trombose subaguda.

Quando estratificado pela apresentação clínica, a mortalidade nos quadros estáveis foi de 1,6\% (4/257)

TABELA 2

Indicação clínica dos procedimentos

\begin{tabular}{lc}
\hline Variável & $\mathbf{n}=\mathbf{5 1 8}$ \\
\hline Síndromes estáveis, n (\%) & $257(49,6)$ \\
Assintomáticos & $91(17,5)$ \\
Angina estável típica & $89(17,2)$ \\
Equivalente isquêmico & $55(10,6)$ \\
Angina atípica & $22(4,3)$ \\
Síndromes coronárias agudas, n $(\%)$ & $261(50,4)$ \\
SCASST & $153(58,6)$ \\
IAMCST & $97(37,2)$ \\
Angina pós-infarto & $11(4,2)$
\end{tabular}

IAMCST = infarto agudo do miocárdio com supradesnivelamento do segmento ST; $\mathrm{n}=$ número de pacientes; SCASST = síndrome coronária aguda sem supradesnivelamento do segmento ST. 
TABELA 3 Características angiográficas

\begin{tabular}{lc}
\hline Variável & $\begin{array}{c}\mathbf{n}=\mathbf{5 1 8} \text { pacientes/ } \\
\mathbf{8 7 6} \text { vasos/890 lesões }\end{array}$ \\
\hline Multiarteriais, n (\%) & $158(30,6)$ \\
Vaso-alvo, n (\%) & $291(33,2)$ \\
DA & $292(33,3)$ \\
CD & $215(24,6)$ \\
Cx & $10(1,2)$ \\
TCE & $67(7,7)$ \\
Enxertos venosos ou arteriais & $648(74)$ \\
Lesões tipo B2/C, $\mathrm{n}(\%)$ & $98(11,2)$ \\
Bifurcação, n (\%) & $103(19,9)$ \\
Calcificação moderada/ & $192(22)$ \\
acentuada, $\mathrm{n}(\%)$ & $114(13)$ \\
Lesões longas (> 20 mm), n (\%) & $61(7)$ \\
Trombo visível, $\mathrm{n}(\%)$ & $9(1)$ \\
Placas rotas/ulceradas, $\mathrm{n}(\%)$ & \\
Ectasia e/ou aneurisma, $\mathrm{n}(\%)$ & \\
\hline CD = coronária direita; Cx = circunflexa; DA = descenden- \\
te anterior; TCE = tronco de coronária esquerda. \\
\hline
\end{tabular}

TABELA 4 Características dos procedimentos

\begin{tabular}{|c|c|}
\hline Variável & $\begin{array}{c}n=518 \text { pacientes/ } \\
876 \text { vasos/890 lesões }\end{array}$ \\
\hline Diâmetro da estenose pré, \% & $81,4 \pm 13,9$ \\
\hline \multicolumn{2}{|l|}{ Fluxo TIMI pré, n (\%) } \\
\hline 0 ou 1 & $141(16,1)$ \\
\hline 2 ou 3 & $734(83,9)$ \\
\hline Stents implantados, n (\%) & $846(95)$ \\
\hline Relação stent/paciente & 1,6 \\
\hline Stents farmacológicos, n (\%) & $314(37,1)$ \\
\hline Diâmetro do stent, mm & $3,01 \pm 0,53$ \\
\hline Comprimento do stent, $\mathrm{mm}$ & $23,2 \pm 7$ \\
\hline \multicolumn{2}{|l|}{ Tipos de intervenção, n (\%) } \\
\hline ICP primária & $78(15)$ \\
\hline ICP de resgate & $13(2,5)$ \\
\hline ICP de urgência & $63(12,1)$ \\
\hline ICP eletiva/ad hoc & $365(70,4)$ \\
\hline $\begin{array}{l}\text { Uso de inibidores da } \\
\text { glicoproteína Ilb/IIla, n (\%) }\end{array}$ & $144(27,8)$ \\
\hline \multicolumn{2}{|l|}{ Fluxo TIMI pós, n (\%) } \\
\hline 0 e 1 & $3(0,3)$ \\
\hline 2 e 3 & $873(99,6)$ \\
\hline Sucesso do procedimento, $\mathrm{n}(\%)$ & $485(93,6)$ \\
\hline
\end{tabular}

e nas SCAs foi de $4,2 \%(11 / 261)$. No grupo do IAMCST, 13 pacientes $(13,4 \%)$ estavam em Killip III/IV, dos quais 4 faleceram, significando mortalidade de 30,8\% nesse grupo de pacientes com grave repercussão hemodinâmica.

$\mathrm{Na}$ análise multivariada, a presença de hipertensão arterial [odds ratio (OR) 4,48, intervalo de confiança de 95\% (IC 95\%) 1,32-15,16; P = 0,016) e de diabetes (OR 7,24, IC 95\% 1,69-31,05; $\mathrm{P}=0,008)$ foram as variáveis que melhor explicaram a presença de ECCAM hospitalares (Tabela 6).

\section{DISCUSSÃO}

Neste estudo, fizemos um levantamento retrospectivo de dados sobre ICP, acumulados prospectivamente ao longo de 5 anos do início da atividade do serviço de cardiologia intervencionista inserido em um hospital privado com tradição cardiológica clínica e cirúrgica, localizado em grande centro fora da capital de São Paulo.

TABELA 5

Desfechos clínicos na fase hospitalar

\begin{tabular}{lc}
\hline Variável & $\mathbf{n}=\mathbf{5 1 8}$ \\
\hline ECCAM, n (\%) & $33(6,4)$ \\
Óbito total, n (\%) & $15(2,9)$ \\
Síndromes estáveis & 4 \\
Síndromes agudas & 11 \\
$\quad$ SCASST & 5 \\
IAMCST & 6 \\
AVC, n (\%) & $4(0,8)$ \\
Infarto do miocárdio, n (\%) & $18(3,5)$ \\
Reintervenção, n (\%) & $4(0,8)$ \\
Trombose de stent, n (\%) & $5(0,97)$ \\
Aguda & 4 \\
Subaguda & 1 \\
\hline AVC = acidente vascular cerebral; ECCAM = eventos cardía- \\
cos e cerebrovasculares adversos maiores; IAMCST = infarto \\
agudo do miocárdio com supradesnivelamento do segmento \\
ST; SCASST = síndrome coronária aguda sem supradesni- \\
velamento do segmento ST.
\end{tabular}

TABELA 6

Preditores independentes de eventos cardíacos e cerebrovasculares adversos maiores hospitalares

\begin{tabular}{lccc} 
Fatores selecionados & OR & IC de 95\% & Valor de $\mathbf{P}$ \\
\hline Hipertensão arterial & 4,48 & {$[1,32 ; 15,16]$} & 0,016 \\
Diabetes & 7,24 & {$[1,69 ; 31,05]$} & 0,008
\end{tabular}

IC $95 \%$ = intervalo de confiança de $95 \%$; OR = odds ratio. 
Ao se comparar os dados demográficos, clínicos e angiográficos, observa-se que nossa população é semelhante, em muitos aspectos, à de outros estudos nacionais, à exceção da média de idade, que se mostrou maior nesta amostra: $65,7 \pm 12,2$ anos comparativamente a $61 \pm 11$ anos no estudo de Neri-Souza et al. ${ }^{8}$, a 60,6 $\pm 10,9$ anos no estudo de Abelin et al. ${ }^{9}$, e a $62 \pm 11,2$ anos no registro do biênio 2003-2004 da Central Nacional de Intervenções Cardiovasculares (CENIC) da Sociedade Brasileira de Hemodinâmica e Cardiologia Intervencionista. ${ }^{10}$ Chama a atenção o fato de que um terço dos pacientes tinha idade $\geq 75$ anos, e a idade é preditora independente de ECCAM em vários estudos e registros. ${ }^{11,12}$

Quanto aos fatores de risco para aterosclerose, a hipertensão arterial foi o mais frequente, incidindo em $72,4 \%$ da população do estudo, estando em consonância com a literatura ${ }^{5,7,10}$ e constituindo-se, nesta análise, em preditor de ECCAM, ao lado do diabetes melito.

No Brasil, a prevalência de obesidade é de cerca de $8 \%$ para os homens e de $12,4 \%$ para mulheres. ${ }^{13}$ A frequência de obesidade (acima de 20\%), nesta amostra, chama a atenção para sua importância no contexto da doença arterial coronária, em que exerce inter-relação direta na gênese e no agravamento de hipertensão arterial, diabetes e dislipidemia. ${ }^{14}$

Quanto à apresentação clínica, metade de nossas intervenções foi realizada no contexto de algum tipo de SCA, fato que comprova a importância crescente da atuação da cardiologia intervencionista nesse cenário. Embasam esse fato as comprovações de que no IAMCST a ICP primária tem mostrado expressiva taxa de sucesso e redução de mortalidade, superando a terapêutica trombolítica isolada; e de que no IAMSST, a estratégia invasiva precoce tem sido igualmente mais efetiva na redução de ECCAM. ${ }^{3,4} \mathrm{O}$ expressivo contingente de pacientes com quadros coronários agudos, por si só, já justifica a necessidade de um hospital de tradição cardiológica possuir em suas instalações um laboratório de cardiologia intervencionista equipado e com profissionais capacitados no atendimento de pacientes com SCA em suas diferentes formas de apresentação.

Despertou a atenção a frequência de pacientes que, mesmo em uso de pelo menos um antiagregante (aspirina ou tienopiridínico de primeira geração) apresentavam quadro de SCA, 52,4\% com IAMCST e 58,7\% com SCASST. Falhas no uso dessas medicações, complexidade clínica, má resposta aos antiagregantes e a importância de outras vias de ativação plaquetária além das vias da ciclo-oxigenase e dos receptores de adenosina P2Y12 poderiam estar entre as possíveis justificativas causais desses achados. ${ }^{15}$

Além da complexidade clínica, angiograficamente um terço dos pacientes eram portadores de doença multiarterial e apresentavam predomínio (74\%) de lesões complexas B2 e C. Com o desenvolvimento tecnológico, com balões e stents de baixo perfil, boa flexibilidade, alto desempenho e ampla disponibilização de diferentes diâmetros e extensões, fios-guia personalizados para diferentes cenários (tortuosidades, oclusões totais), novos dispositivos (como cutting-balloon) e técnicas ateroablativas, eficiente farmacoterapia adjunta, excelente qualidade de imagem radiológica e suporte hemodinâmico, o tratamento intervencionista de pacientes angiográfica e clinicamente mais complexos tem se tornado uma realidade diária dos laboratórios de hemodinâmica, desde que adequadamente equipados e operados. ${ }^{16} \mathrm{~A}$ alta taxa de uso de stents neste estudo está condizente com o avanço tecnológico, sendo pouco mais de um terço desses stents farmacológicos. Esses dispositivos foram implantados preferencialmente em pacientes com lesões complexas e diabetes melito, mais em consequência das restrições de financiamento por parte do sistema de saúde suplementar que por evidências clínicas atuais. ${ }^{17}$

O sucesso do procedimento de 93,6\%, mesmo em um cenário que inclui pacientes com complexidades clínicas e angiográficas, está adequado ao observado na literatura. A taxa geral de óbito foi de 2,9\%, influenciada principalmente pela sua maior ocorrência nas síndromes coronárias agudas, em especial no IAMCST, que foi de 6,2\% (6 óbitos em 97 casos). Observa-se, no entanto, que dois terços desses óbitos (4 em 6) se deram em pacientes em grupo funcional Killip III/IV, importante preditor de mortalidade. ${ }^{18}$ As taxas de AVC $(0,8 \%)$, de infarto pós-procedimento (3,5\%), de reintervenção $(0,9 \%)$ e de trombose aguda e subaguda de stents $(0,97 \%)$ foram baixas e equivalentes às constantes na literatura. ${ }^{19-21}$

\section{Limitações do estudo}

Reconhecemos a análise retrospectiva dos dados, sua realização em um único centro e a ausência de seguimento tardio como limitações deste estudo.

\section{CONCLUSÕES}

O sucesso do procedimento e a baixa ocorrência de desfechos clínicos desfavoráveis demonstram a efetividade e a segurança da ICP no atendimento de pacientes, mesmo em situações de maior complexidade, tratados em um novo serviço de cardiologia intervencionista inserido em hospital privado localizado em grande centro fora da capital de São Paulo.

\section{CONFLITO DE INTERESSES}

Os autores declaram não haver conflito de interesses relacionado a este manuscrito.

\section{REFERÊNCIAS}

1. Serruys PW, Morice MC, Kappetein AP, Colombo A, Holmes DR, Mack MJ, et al.; SYNTAX Investigators Percutaneous coronary intervention versus coronary-artery bypass grafting 
for severe coronary artery disease. N Engl J Med. 2009;360(10): 961-72.

2. Cohen DJ, Van Hout B, Serruys PW, Mohr FW, Macaya C, den Heijer $\mathrm{P}$, et al. Quality of life after $\mathrm{PCl}$ with drug-eluting stents or coronary-artery bypass surgery. N Engl J Med. 2011; 364(11):1016-26.

3. Westerhout CM, Bonnefoy E, Welsh RC, Steg PG, Boutitie $F$, Armstrong PW. The influence of time from symptom onset and reperfusion strategy on 1-year survival in ST-elevation myocardial infarction: a pooled analysis of an early fibrinolytic strategy versus primary percutaneous coronary intervention from CAPTIM and WEST. Am Heart J. 2011;161(2):283-90.

4. Brasil. Ministério da Saúde. DATASUS. Cadernos de Informação de Saúde [Internet]. Brasília; 2010 [citado 2011 out. 25]. Disponível em: http://tabnet.datasus.gov.br/tabdata/cadernos/ BR/Brasil_GeralBR.xls

5. Mattos LA, Lemos Neto PA, Rassi A Jr, Marin-Neto JA, Sousa AGMR, Devito FS, et al. Diretrizes da Sociedade Brasileira de Cardiologia - Intervenção Coronária Percutânea e Métodos Adjuntos Diagnósticos em Cardiologia Intervencionista (II Edição - 2008). Rev Bras Cardiol Invasiva. 2008;16 Supl 2:9-88.

6. Smith SC Jr, Feldman TE, Hirshfeld JW Jr, Jacobs AK, Kern MJ, King SB $3^{\text {rd }}$, et al. ACC/AHA/SCAI 2005 guideline update for percutaneous coronary intervention: a report of the American College of Cardiology/American Heart Association Task Force on Practice Guidelines (ACC/AHA/SCAI Writing Committee to Update 2001 Guidelines for Percutaneous Coronary Intervention). Circulation. 2006;113(7):e166-286.

7. TIMI Study Group. The Thrombolysis in Myocardial Infarction (TIMI) trial. Phase I findings. N Engl J Med. 1985;312(14): 932-6.

8. Neri-Souza AJ, Aguiar BM, Coelho AB, Nascimento AJ, Oliveira Junior WS, Godinho AG, et al. Preditores independentes de resultados intra-hospitalares pós-implante de stent coronariano. Arq Bras Cardiol. 2006;87(4):429-38.

9. Abelin AP, Quadros AS, Zanettini MT, Leboute FC, Yordi LM, Cardoso CR, et al. Doze anos de experiência com implante de stents coronários em 5.284 pacientes. Rev Bras Cardiol Invasiva. 2009;17(3):346-51.

10. Mangioni JA. Intervenção coronária percutânea no Brasil. Quais são os nossos números? Rev Bras Cardiol Invasiva. 2006;14(3):267-72.

11. Yan BP, Gurvitch R, Duffy SJ, Clark DJ, Sebastian M, New $G$, et al. An evaluation of octogenarians undergoing percutaneous coronary intervention from the Melbourne Inter- vention Group registry. Catheter Cardiovasc Interv. 2007;70(7): 928-36.

12. Merchant FM, Weiner RB, Rao SR, Lawrence R, Healy JL, Pomerantsev E, et al. In-hospital outcomes of emergent and elective percutaneous coronary intervention in octogenarians. Coron Artery Dis. 2009;20(2):118-23.

13. Guimarães HP, Avezum A, Piegas LS. Obesidade abdominal e síndrome metabólica. Rev Soc Cardiol Estado de São Paulo. 2006;16(1):41-7.

14. Wilson PW, D'Agostino RB, Sullivan L, Parise H, Kannel WB. Overweight and obesity as determinants of cardiovascular risk: the Framingham experience. Arch Intern Med. 2002;162(16): 1867-72.

15. White HD. Oral antiplatelet therapy for atherothrombotic disease: current evidence and new directions. Am Heart J. 2011;161(3):450-61.

16. Epstein AJ, Polsky D, Yang F, Yang L, Groeneveld PW. Coronary revascularization trends in the United States, 20012008. JAMA. 2011;305(17):1769-76.

17. Wijns W, Kolh P, Danchin N, Di Mario C, Falk V, Folliguet $\mathrm{T}$, et al. Guidelines on myocardial revascularization. The Task Force on Myocardial Revascularization of the European Society of Cardiology (ESC) and the European Association for Cardio-Thoracic Surgery (EACTS). Developed with the special contribution of the European Association for Percutaneous Cardiovascular Interventions (EAPCI). Eur Heart J. 2010;31(20):2501-55.

18. Lee KL, Woodlief LH, Topol EJ, Weaver WD, Betriu A, Col J, et al. Predictors of 30-day mortality in the era of reperfusion for acute myocardial infarction. Results from an international trial of 41,021 patients. GUSTO-I Investigators. Circulation. 1995;91(6):1659-68.

19. Daemen J, Boersma E, Flather M, Booth J, Stables R, Rodriguez $A$, et al. Long-term safety and efficacy of percutaneous coronary intervention with stenting and coronary artery bypass surgery for multivessel coronary artery disease: a meta-analysis with 5-year patient-level data from ARTS, ERACI-II, MASS-II, and SoS Trials. Circulation. 2008;118(11):1146-54.

20. Ritchie JL, Maynard C, Every NR, Chapko MK. Coronary artery stent outcomes in a Medicare population: less emergency bypass surgery and lower mortality rates in patients with stents. Am Heart J. 1999;138(3 Pt 1):437-40.

21. Singh M, Rihal CS, Gersh BJ, Lennon RJ, Prasad A, Sorajja $\mathrm{P}$, et al. Twenty-five-year trends in in-hospital and long term outcome after percutaneous coronary intervention: a singleinstitution experience. Circulation. 2007;115(22):2835-41. 